\title{
중 \\ Efectos de la biodiversidad en el control biológico dentro de los agroecosistemas
}

\author{
Jonhy Alexander Hidalgo Martín ${ }^{1}$ Álvaro Acevedo Osorio²
}

Recibido: agosto 16 de 2012 Aprobado: octubre 12 de 2012

\section{RESUMEN}

El control biológico de plagas en los agroecosistemas se ve favorecido por los policultivos (cultivos asociados, sistemas agroforestales) y de vegetación no cultivada (corredores biológicos, plantas arvenses), ya que estos sirven como hábitats alternos para las poblaciones de insectos benéficos. Durante las últimas décadas, los sistemas agrícolas se han transformado debido al establecimiento de monocultivos que han homogenizado los paisajes agrícolas; así se han generado condiciones desfavorables para los enemigos naturales de plagas al no encontrar en los monocultivos fuentes diversas para su alimentación como polen, presas, o sitios de reproducción y de refugio. Como consecuencia, las poblaciones de insectos plaga han aumentado dramáticamente en los monocultivos causando daños de importancia económica. Para su control se están empleando productos de síntesis química con diversas dosis y grados de toxicidad, lo que provoca grandes costos ambientales (impacto negativo sobre la fauna silvestre, los polinizadores, los enemigos naturales, la pesca, la calidad del agua) y costos sociales (envenenamiento de trabajadores, contaminación de alimentos, enfermedades humanas) no suficientemente considerados. Debido a estos problemas generados por la homogenización de los cultivos y la intensificación en el uso de agrotóxicos, se ha incrementado la valoración del uso adecuado de la biodiversidad como aspecto indispensable para restablecer el control biológico o natural; la agroecología juega un papel fundamental en este objetivo mediante el diseño de agroecosistemas diversificados que aumentan los sinergismos entre los componentes clave del agroecosistema.

Palabras clave: control biológico, biodiversidad, agroecosistema.

\section{ABSTRACT}

Biological control of pests in agro-ecosystems is favored by the establishment of poly-cultivation (mixed cropping, agro-forestry systems) and cultivated vegetation (biological corridors, plants weeds), as they serve as alternative habitats that permit beneficial insect populations. In recent decades, agricultural systems was trnsformed due to the establishment of mono-cultivation that has homogenized landscapes generating adverse conditions for natural enemies of pests as they don't find sources of food like pollen, dams, or breeding sites and shelter in mono-cultivation. Consequently, the insect pest populations have increased dramatically in single cultivation causing damage of economic kind. To control this, there is the use of synthetic chemical products with various doses and degrees of toxicity which implying high environmental costs (negative impact on wildlife, pollinators, natural enemies, fisheries, water quality) and social costs (workers poisoning, food contamination, human diseases) is not considered enough. Because of these problems caused by the homogenization of crops and the intensification of pesticide use, there has been an increased value of proper use of biodiversity as a cornerstone to restore the natural or biological control. Agro-ecology plays a crucial role in this objective through a diversified agro-ecosystem design that increases synergisms among key components of the agro-ecosystem.

Keywords: Biological control, biodiversity, agro-ecosystem.

1 Estudiante VIII Semestre. Programa Ingeniería Agroecológica. Corporación Universitaria Minuto de Dios.

2 Máster en Agroecología; candidato a doctor en Agroecología. Docente - Investigador. Grupo AGROECO Y GESTIÓN AMBIENTAL. 


\section{INTRODUCCIÓN}

En los sistemas agrícolas la biodiversidad está constituida por especies que interactúan y proporcionan servicios ecosistémicos como reciclaje de nutrientes, control biológico, polinización, entre otros. El control biológico en los agroecosistemas se ve favorecido por la conservación de la biodiversidad. De acuerdo con McNeely et al. (1990), Altieri y Nicholls (2007) la biodiversidad en los agroecosistemas incluye componentes tan variados como son los cultivos, arvenses, artrópodos y microorganismos asociados, así como los factores de situación geográfica, climáticos, edáficos, humanos y socioeconómicos. En la naturaleza, la abundancia y la distribución de organismos está regulada por la interacción entre los factores abióticos (clima, disponibilidad de refugios) y los factores bióticos (enemigos naturales).

La agricultura tecnificada implica la simplificación de la biodiversidad, ya que se establecen monocultivos para obtener una producción artificial que requiere de una constante intervención humana (Altieri, 1992; Bustamante y Grez, 1995). Generalmente los costos ambientales y sociales se manifiestan con aparición recurrente de plagas, contaminación de aguas, salinización y erosión de suelos, la extinción local o regional de especies, la pérdida de recursos genéticos, el aumento en la ocurrencia de plagas, la disminución en la polinización de cultivos y la alteración de los procesos de formación y mantenimiento de los suelos, entre otros (Altieri, 1992). El propósito de este artículo es analizar la importancia de la conservación de la biodiversidad dentro y alrededor de los sistemas productivos, en tanto se determina la presencia de diversos insectos en el agroecosistema que prestan servicios a cultivos tanto en la polinización como en el control biológico de insectos plaga.

\section{BIODIVERSIDAD Y CONTROL BIOLOGICO}

\section{Consecuencias de la simplificación de la biodiversidad}

La inestabilidad de los agroecosistemas se manifiesta a través del incremento de los problemas relacionados con insectos plaga, ligados a la expansión de monocultivos a expensas de la vegetación natural, disminuyendo la diversidad del hábitat local (Altieri y Letourneau, 1982; Flint y Roberts, 1988). Las comunidades de plantas que se modifican para satisfacer las necesidades especiales de los humanos quedan inevitablemente sujetas a daños por plagas. Generalmente, mientras más intensa sea la modificación de estas comunidades más abundantes y serios son los problemas de plagas. Según Nicholls (2006) una con- secuencia de esta tendencia es que la cantidad total de hábitat disponible para insectos benéficos está decreciendo a tasas alarmantes. En la medida en que se homogeniza el paisaje y aumenta la alteración en el ambiente, este se torna cada vez más desfavorable para los enemigos naturales. Las implicaciones de la pérdida de hábitat para el control biológico de plagas pueden ser serias, dadas las evidencias que demuestran un incremento de plagas en los paisajes agrícolas homogéneos (Altieri y Letourneau, 1982).

De acuerdo con Altieri y Nicholls (2002) en monocultivos los fitófagos logran una mayor colonización, mayor reproducción, mayor tiempo de permanencia en el cultivo, menor dificultad para encontrar el cultivo y menor mortalidad debida a enemigos naturales. Los monocultivos son ambientes difíciles para inducir una operación eficiente en contra de enemigos naturales, debido a que estos carecen de recursos adecuados para el desempeño óptimo de depredadores y parasitoides porque, en general, se usan prácticas que afectan negativamente el control biológico. No obstante, Nicholls (2008) afirma que el control biológico de plagas dejó de practicarse con la generalización de la lucha química como medida de control de plagas, enfermedades y arvenses hasta que, por los diversos problemas que ocasionó el uso intensivo de plaguicidas, ganó de nuevo terreno como alternativa en el manejo de la salud vegetal.

Altieri y Nicholls (2012) sostienen que debido a la falta de mecanismos de regulación ecológica los monocultivos son altamente dependientes de pesticidas; en los últimos 50 años, el uso de pesticidas se ha incrementado dramáticamente en todo el mundo y ahora asciende 2,6 millones de toneladas de pesticidas por año, con un valor anual en el mercado global que supera 25 mil millones de dólares. Nada más en Estados Unidos se usan anualmente 324 millones de kilogramos de 600 diferentes tipos de pesticidas, con enormes costos indirectos en el ambiente (impacto sobre la fauna silvestre, los polinizadores, los enemigos naturales, la pesca, la calidad de agua, etc.) y costos sociales (envenenamiento de trabajadores y enfermedades humanas), estos valores ascienden a cerca de 8 mil millones de dólares cada año. Además, 540 especies de artrópodos han desarrollado resistencia a más de mil diferentes tipos de pesticidas que ahora son incapaces de controlar químicamente a esas plagas; esto también se evidencia en los demás países del mundo en donde se ha realizado aplicación de productos de síntesis química en dosis indiscriminadas.

\section{Arreglos espaciales y temporales}

De acuerdo con Van Den Bosch y Telford (1964) existen varios factores ambientales que afectan la diversidad, 
abundancia y actividad de parasitoides y depredadores en los agroecosistemas: condiciones microclimáticas, disponibilidad de alimentos (agua, polen, presas, etc.), recursos del hábitat (sitios de reproducción, refugio, etc.), competencia interespecífica y presencia de otros organismos (hiperparásitos, depredadores, etc.). Los efectos de cada uno de estos factores varían de acuerdo con el arreglo espacio temporal de los cultivos y a la intensidad de manejo; debido a que estos atributos afectan la heterogeneidad ambiental de los agroecosistemas.

En los agroecosistemas modernos la evidencia experimental sugiere que la biodiversidad puede ser utilizada para mejorar el control de plagas (Altieri y Letourneau, 1984; Andow, 1991). Varios estudios han puesto de manifiesto que es posible estabilizar las comunidades de insectos en los agroecosistemas, diseñando sistemas agrícolas diversos, tales como policultivos, sistemas agroforestales y cultivos de cobertura que apoyen a las poblaciones de enemigos naturales o que tengan efectos sobre los fitófagos (Perrin, 1980; Risch et al, 1983). Según Altieri y Nicholls (2002) el tipo y la abundancia de biodiversidad dependen de la estructura y manejo del agroecosistema. Southwood y Way (1970) señalaron que, en general, el nivel de biodiversidad de insectos en los agroecosistemas depende de cuatro características principales: a) la diversidad de vegetación en y alrededor del agroecosistema; b) la durabilidad del cultivo dentro del agroecosistema; c) la intensidad del manejo y d) el aislamiento del agroecosistema con respecto a la vegetación natural.

\section{Efecto sobre la regulación de organismos indeseables}

Altieri (1991) afirma que una de las razones más importantes para mantener o incrementar la biodiversidad natural es el hecho de que ésta proporciona una gran variedad de servicios ecológicos. Hay muchas prácticas y diseños agrícolas que tienen el potencial de estimular las funciones de la biodiversidad y otras que las afectan negativamente. La idea es aplicar las mejores prácticas de manejo para favorecer o regenerar el tipo de biodiversidad que puede contribuir a la sostenibilidad del agroecosistema. De acuerdo con Altieri y Nicholls (2007) una de las funciones de los agroecólogos es fomentar esas prácticas agrícolas que incrementan la abundancia y la diversidad de organismos benéficos presentes sobre el suelo o el subsuelo y que, por lo tanto, ofrezcan importantes servicios ecológicos a los agroecosistemas. El desafío de diseñar tales arquitecturas solamente se podrá enfrentar estudiando las relaciones entre la diversificación de la vegetación y la dinámica poblacional de los herbívoros y sus enemigos naturales asociados en agroecosistemas particulares.
Uno de los servicios ecológicos es la regulación de la abundancia de organismos indeseables a través de la depredación, el parasitoidismo y la competencia (Altieri, 1994). Probablemente cada población de insectos en la naturaleza es atacada en alguna medida por uno o más enemigos naturales. Así, depredadores, parasitoides y patógenos actúan como agentes de control natural que, cuando son adecuadamente manejados pueden determinar la regulación de poblaciones de herbívoros en un agroecosistema particular. Esta regulación ha sido llamada control biológico y fue definido por DeBach (1964), Botto (2002) y Nicholls (2008) como la acción de enemigos naturales y microorganismos para mantener la densidad de la población de un organismo plaga a un promedio menor de la que ocurriría en su ausencia.

\section{Aumento de la biodiversidad}

A pesar de que los enemigos naturales varían en su respuesta a la distribución, densidad y dispersión de los cultivos, la evidencia señala que ciertos atributos estructurales del agroecosistema (diversidad vegetal, los niveles de insumos empleados como fertilizantes o en el manejo de plagas y enfermedades) influyen significativamente en la dinámica y diversidad de depredadores y parasitoides (Altieri y Nicholls, 2002). La mayoría de estos atributos se relacionan con la biodiversidad y están sujetos al manejo (por ejemplo, asociaciones y rotaciones de cultivos, presencia de arvenses en floración, diversidad genética, etc.) (Rabb et al., 1976; Altieri, 1994).

En la medida que se homogeneiza el paisaje y aumenta la perturbación del ambiente este se torna cada vez más desfavorable para los enemigos naturales. Las implicaciones de la pérdida de hábitat para el control biológico de plagas pueden ser serias, según las evidencias que demuestran un incremento de plagas en los paisajes agrícolas homogéneos (Altieri y Letourneau, 1982). De acuerdo con Barbosa (1998) datos recolectados en estudios demuestran que hay un incremento de enemigos naturales y un control biológico eficiente en áreas donde permanece la vegetación natural en los bordes de las plantaciones. Landis et al. (2000) afirma que estos hábitats son importantes como sitios de refugio ya que proveen alimento para enemigos naturales en épocas de escasez de plagas en los sistemas productivos, además del polen y el néctar. Según Altieri y Whitcomb (1979) las arvenses y otro tipo de vegetación alrededor de los campos albergan presas y hospedantes para los enemigos naturales, proporcionando así recursos estacionales y cubriendo las brechas en los ciclos de vida de los insectos entomófagos y de las plagas.

Según la teoría agroecológica, el comportamiento óptimo de un agroecosistema depende del nivel de 
interacciones entre los diversos componentes bióticos y abióticos. En el contexto de una biodiversidad funcional es posible iniciar sinergismos que contribuyan a favorecer procesos, al ofrecer servicios ecológicos tales como la activación de la biología del suelo, el ciclaje de nutrientes, el fomento de artrópodos y antagonistas beneficiosos, entre otros (Altieri, 1995; Gliessman, 1998), todos ellos importantes para determinar la sostenibilidad de los agroecosistemas (Altieri y Nicholls, 2007).

\section{Diversificación}

En agroecosistemas diversificados se evidencia que en la medida que se incrementa la diversidad vegetal, la reducción de plagas alcanza un nivel óptimo dependiendo del número de especies vegetales y la combinación de ciertas plantas. Aparentemente, mientras más diverso es el agroecosistema y mientras menos alterada es la diversidad, los nexos tróficos aumentan desarrollándose sinergismos que promueven la estabilidad poblacional insectil (Southwood y Way, 1970). Estos eventos se presentan ya que los insectos benéficos encuentran hábitats alternos en donde se hallan las condiciones óptimas para alimentarse y reproducirse, además estos hábitats les brindan refugio (Van Den Bosch y Telford, 1964; Norris y Kogan, 2005; Nicholls, 2008; Gamboa y Criollo, 2011).

La manipulación de la vegetación natural adyacente a los campos de cultivo puede ser usada para promover el control biológico, ya que la supervivencia y actividad de varios enemigos naturales depende de los recursos ofrecidos por la vegetación contigua al campo. Los cercos vivos y otros aspectos del paisaje han recibido gran atención debido a sus efectos en la distribución y abundancia de artrópodos en las áreas adyacentes a los sistemas productivos (Fry, 1995). En general, se reconoce la importancia de la vegetación natural alrededor de los sistemas productivos como reserva de enemigos naturales de plagas (Van Emden, 1965). Muchos estudios han documentado el movimiento de enemigos naturales desde los márgenes hacia el centro de los cultivos, demostrando un mayor nivel de control biológico en hileras de cultivos adyacentes a vegetación natural (Pickett y Bugg, 1998, Thies y Tscharntke, 1999).

\section{Agroecología y biodiversidad}

A medida que progresa la modernización agrícola, los principios ecológicos se ignoran o desestiman continuamente: como resultado, los agroecosistemas modernos son inestables. Los desequilibrios se manifiestan como brotes recurrentes de plagas y enfermedades en numerosos cultivos, en la salinización y erosión del suelo, contaminación de aguas y otros problemas am- bientales. Estos son claros signos de que la estrategia del control de plagas y enfermedades que se basa en la utilización de productos de síntesis química ha llegado a su límite (Nicholls, 2008). El empeoramiento de los problemas de plagas y enfermedades se relaciona experimentalmente con la expansión de los monocultivos a expensas de la diversidad vegetal, la cual constituye un componente esencial del paisaje que proporciona servicios ecológicos claves para asegurar la protección de cultivos (Altieri y Letourneau, 1982). En este sentido es necesario plantear una estrategia alternativa, que se base en el uso de los principios ecológicos para aprovechar al máximo los beneficios de la biodiversidad en la agricultura y por esta razón, en la actualidad el control biológico se considera una pieza fundamental e indispensable en cualquier estrategia de agricultura sostenible con base agroecológica.

Desde una perspectiva de manejo, el objetivo de la agroecología es proveer ambientes balanceados, rendimientos sustentables, una fertilidad del suelo biológicamente obtenida y una regulación natural de las plagas a través del diseño de agroecosistemas diversificados y el uso de tecnologías de bajos insumos (Gliessman, 1998). Los agroecólogos están ahora reconociendo que los policultivos, la rotación de cultivos, los corredores biológicos, la agroforestería y otros métodos de diversificación imitan los procesos ecológicos naturales, y que la sustentabilidad de los agroecosistemas complejos se basa en los modelos ecológicos que ellos siguen. Mediante el diseño de sistemas de cultivo que imiten la naturaleza puede hacer un uso óptimo de la luz solar, de los nutrientes del suelo y de la lluvia (Pret, 1994).

El manejo agroecológico debe tratar de optimizar el reciclado de nutrientes y de materia orgánica, cerrar los flujos de energía, conservar el agua, el suelo y balancear las poblaciones de plagas y enemigos naturales. La estrategia explota las complementariedades y sinergismos que resultan de varias combinaciones de cultivos, árboles y animales, en arreglos espaciales y temporales diversos (Altieri, 1994).

La agroecología provee el conocimiento y la metodología necesaria para desarrollar una agricultura que sea, por un lado, ambientalmente adecuada y por el otro lado, altamente productiva, socialmente equitativa y económicamente viable (Altieri, 1994). A través de la aplicación de los principios agroecológicos, el desafío básico de la agricultura sustentable de hacer un mejor uso de los recursos internos puede ser fácilmente alcanzado, minimizando el uso de insumos externos y preferentemente generando los recursos internos más eficientemente a través de las estrategias de diversificación que aumenten los sinergismos entre los componentes clave del agroecosistema. 


\section{CONCLUSIONES}

Los agroecosistemas se ven beneficiados por la conservación de la biodiversidad silvestre y los arreglos de alta diversidad de cultivos, favoreciendo la permanencia de los enemigos naturales de organismos parásitos de los cultivos; las especies plantadas muchas veces sirven como hábitats alternos para la hibernación y alimentación de enemigos naturales ya que requieren de polen o néctar, además que albergan presas para los enemigos naturales cubriendo épocas de ausencia de insectos indeseables (entomófagos y plagas).

Los arreglos espacio temporales en agroecosistemas son de suma importancia para la conservación de la biodiversidad debido a que en estas áreas se encuentran diversos servicios ecosistémicos, entre ellos el control biológico que se ve favorecido por la alta oferta de fuentes alternativas de hábitat en donde pueden sobrevivir y alimentarse de otras especies durante la ausencia de la población de la cual se alimentan.

La homogenización de los sistemas productivos conduce a una inestabilidad de los agroecosistemas, debido a que las poblaciones de insectos indeseables encuentran mayor disponibilidad de alimentación y las poblaciones de enemigos naturales tienden a desaparecer ya que no hallan disponibilidad de fuentes alternativas de alimentación, además las aplicaciones de productos de síntesis química las afectan.

\section{REFERENCIAS BIBLIOGRÁFICAS}

1. Altieri, M. (1991). Traditional fam-iing in Latin America. The Ecologist 21: 93-96.

2. Altieri, M. (1992). El rol ecológico de la biodiversidad en agroecosistemas. Agroecología y Desarrollo. Chile: Clades. Recuperado de http: / / www.clades.cl/revistas/4/rev4art1.htm

3. Altieri, M. (1994). Biodiversity and pest management in agroecosystems. New York; Haworth Press, 185.

4. Altieri, M. (1995). Agroecology: the Science of Sustainable Agriculture. Boulder: Westview Press

5. Altieri, M. (s.f.) Agroecologia: principios y estrategias para diseñar una agricultura que conserva recursos naturales y asegura la soberanía alimentaria. Berkeley: Universidad de California.

6. Altieri, M y Whitcomb, W. (1979). The potential use of weeds in the manipulation of beneficial insects. HortScience 14: 12-18.
7. Altieri, M y Letourneau, D. (1982). Vegetation management and biological control in agroecosystems. Crop Protection 1: 405-430.

8. Altieri, M y Nicholls, C. (2002). Biodiversidad y diseño agroecológico: un estudio de caso de manejo de plagas en viñedos. Manejo Integrado de Plagas y Agroecología (Costa Rica). 65: 50-64.

9. Altieri M y Nicholls, C. (2007). Biodiversidad y manejo de plagas en agroecosistemas. Barcelona: Icaria editorial.

10. Altieri, M y Nicholls, C. (2012). Agroecología: única esperanza para la soberanía alimentaria y la resiliencia socioecológica. Artículo preparado para Conferencia de las Naciones Unidas sobre el Desarrollo Sostenible (Rio+20), con contribución de otros miembros de SOCLA. Río de Janeiro, Brasil.

11. Andow, D. (1991). Vegetational diversity and artropod population response: Annual review of Entomology, 36: 561-586.

12. Barbosa, P. (1998). Conservation Biological Control. Editado por autor. San Diego, Estados Unidos.

13. Botto, E. (2002). Selección de enemigos naturales para su empleo en el control biológico aplicado. Insectario de Investigaciones para Lucha Biológica. INTA. Castelar Argentina.

14. Bustamante, R. y Grez, A. (1995). Consecuencias ecológicas de la fragmentación de los bosques nativos Ciencia. Ambiente y Desarrollo 11(2): 58-63.

15. DeBach, P. (1964). Biological control of insect pests and weeds. Nueva York: Reinhold Publishing Corporation.

16. Flint, M y Roberts, P. (1988). Using crop diversity to manage pest problems: some California examples. American Journal of Alternative Agriculture 3: 164-167.

17. Fry, G. (1995). Landscape ecology of insect movement in arable ecosystems. Ecology and integrated farming systems. Ed. Bristol.

18. Gamboa, L. y Criollo, M. (2011). Forestería análoga y su rol en la recuperación de ecosistemas y el cambio climático. LESIA revista de agroecología. 27 (2): 8-12.

19. Gliessman, S. (1998). Agroecology: ecological processes in agriculture. Michigan: Ann Arbor Press. 
20. Landis, D; Wratten, S; Gurr, G (2000). Habitat management to conserve natural enemies of arthropod pests in agriculture. Annual Review of Entomology 45:175-201.

21. Mcneely, J. Miller, K.; Reid, W.; Mittermeier, R. y Werner, T. (1990). Conserving the world's biological diversity. Washington, D.C: Banco Mundial.

22. Nicholls, C. (2008). Control Bilógico de insectos: un enfoque agroecológico. Medellín: Editorial Universidad de Antioquia.

23. Nicholls, C. (2006). Bases agroecológicas para diseñar e implementar una estrategia de manejo de hábitat para control biológico de plagas. Agroecología. 1: 37- 48.

24. Norris, R. y Kogan, M. (2005). Ecology of interactions between weeds and arthropods. Annual review of entomology 50:479-503.

25. Perrin, R. (1980). The role of environmental diversity in crop protection. Protection Ecology 2: 77-114.

26. Pickett, C y Bugg, R. (1998). Enhancing Biological Control: habitat management to promote natural enemies of agricultural pests. Berkeley: Universidad de California.

27. Pret, J. (1994). Regenerating agriculture. Londres: Earthscan Publications Ltd.
28. Rabb, R; Stinner, R. y R Van den Bosch, R. (1976). Conservation and augmentation of natural enemies. En: Huffaker, C. y Messenger, P. (Eds.), Theory and Practice of Biological Control. Nueva York: Academic Press, 233-253.

29. Risch, S. (1983). Intercropping as a cultural pest control: prospects and limitations. Environ. Management 7: 9-14. York, Academic Press, pp. 233-253.

30. Southwood, T y Way, M. (1970). Ecological background to pest management. En Rabb y Guthrie F. (Eds). Concepts of Pest Management. Raleinh, Carolina: Universidad del Estado de Norte de Carolina, 6-29.

31. Thie, C y Tscharntke, T. (1999). Landscape structure and biological control in agroecosystems. Science 285: 893-895.

32. Van den Bosch, R. y Telford, A. (1964). Environmental modification and biological control. En DeBach, P. (Ed.) Biological Control of Pests and Weeds. Londres: Chapman and Hall. 459-488.

33. Van Emden, H. (1965). The role of uncultivated land in the biology of crop pests and beneficial insects. Scientific Horticulture 17: 121-126. 